\title{
Rates and Risk Factors of Revision Arthroscopy or Conversion to Total Knee Arthroplasty Within 1 Year Following Isolated Meniscectomy
}

\author{
Avinesh Agarwalla, M.D., Anirudh K. Gowd, M.D., Joseph N. Liu, M.D., \\ Nirav H. Amin, M.D., and Brian C. Werner, M.D.
}

\begin{abstract}
Purpose: To identify the rates of and risk factors for revision arthroscopy and conversion to total knee arthroplasty (TKA) within 1 year of isolated meniscectomy. Methods: Humana and Medicare national insurance databases were queried for patients who underwent isolated meniscectomy. Patients who underwent revision arthroscopy or TKA within l year postoperatively were identified by International Classification of Diseases Procedural Codes, Ninth Revision, and Current Procedural Terminology codes. Multivariate binomial logistic regression analysis was used to identify risk factors, and adjusted odds ratios (ORs) and 95\% confidence intervals (Cis) were calculated, with $P<.05$ considered significant. Results: A total of 13,142 patients and 407,888 patients underwent isolated meniscectomy in the Humana and Medicare databases, respectively. Of the patients, $395(3.01 \%)$ and 3,770 patients $(0.92 \%)$ underwent revision arthroscopy, and 629 patients $(4.79 \%)$ and 38,630 patients $(9.47 \%)$ underwent TKA within 1 year of meniscectomy in the Humana and Medicare databases, respectively. Obesity (Humana: $\mathrm{OR}=1.33, P=0.003$; Medicare: $\mathrm{OR}=1.10, P<0.001$ ) and age $<20$ years (Humana: OR $=2.64, P=0.022$ ), 20-29 years (Humana: OR $=3.30, P=0.002$ ), 40-49 years (Humana: OR $=3.80$, $P<0.001$ ), 50-59 years (Humana: $\mathrm{OR}=1.99, P=0.027$ ), and $<64$ years (Medicare: $\mathrm{OR}=1.74, P<0.001)$ were risk factors for revision arthroscopy. Obesity (Humana: OR $=1.64, P<0.001$; Medicare: OR $=1.37, P<0.001$ ), morbid obesity (Medicare: $\mathrm{OR}=1.20, P<0.001$ ), age 70-74 (Medicare: OR $=1.12, P<0.001)$, 75-79 (Medicare: OR $=1.25, P<$ 0.001 ), 80-84 (Medicare: $\mathrm{OR}=1.20, P<0.001$ ), and concomitant osteoarthritis (Humana: OR $=1.42, P<0.001$; Medicare: $\mathrm{OR}=1.46, P<0.001)$ were risk factors for conversion to TKA. Conclusions: Medicare and Humana databases showed that $0.92 \%-3.01 \%$ and $4.79 \%-9.47 \%$ of patients undergo revision arthroscopy or conversion to TKA within a year of isolated meniscectomy. Obesity was a risk factor for early revision arthroscopy and conversion to TKA, whereas concomitant osteoarthritis was a risk factor for conversion to TKA. Level of Evidence: Level III, retrospective comparative trial.
\end{abstract}

M eniscal injuries are the most common intraarticular knee injuries in the United States and represent a common cause of knee pain and functional impairment. $^{1-3}$ Meniscal injuries in the setting of

From the Department of Orthopaedic Surgery, Westchester Medical Center, Valhalla, New York, U.S.A. (A.A.); Department of Orthopaedic Surgery, Wake Forest University Baptist Medical Center, Winston-Salem, North Carolina, U.S.A. (A.K.G.); Department of Orthopaedic Surgery, Loma Linda University Medical Center, Loma Linda, California, U.S.A. (J.N.L., N.H.A.), and Department of Orthopaedic Surgery, University of Virginia Health System, Charlottesville, Virginia, U.S.A. (B.C.W.).

The authors report the following potential conflicts of interest or sources of funding: N.H.A. receives research support grants from Arthrex, Stryker, Trice Medical, Zimmer, Smith $\theta$ Nephew, and Pacira, outside the submitted work; is a paid presenter or speaker for Depuy, Flexion, Myoscience, Pacira, Smith $\theta$ Nephew, Trice Medical, and Zimmer, outside the submitted work; and is a paid consultant for Smith $\theta$ Nephew, outside the submitted work. B.C.W. is a board or committee member for the American Orthopaedic Society for Sports Medicine and American Shoulder and Elbow Surgeons, outside the submitted osteoarthritis can be treated conservatively. ${ }^{4}$ However, arthroscopic meniscectomy or debridement is a treatment modality for patients with continued symptomatology who have failed nonoperative management. ${ }^{5-7}$

work; receives research support grants from Arthrex, Biomet, and Integra LifeScience, outside the submitted work; and is a paid presenter or speaker for Arthrex, outside the submitted work. Full ICMJE author disclosure forms are available for this article online, as supplementary material.

Received April 27, 2019; accepted April 17, 2020.

Address correspondence to Brian C. Werner, M.D., Department of Orthopaedic Surgery, University of Virginia, PO Box 800159, Charlottesville, VA 22908,U.S.A.E-mail: BCW4X@hscmail.mcc.virginia.edu

(C) 2020 THE AUTHORS. Published by Elsevier Inc. on behalf of the Arthroscopy Association of North America. This is an open access article under the CC BY-NC-ND license (http://creativecommons.org/licenses/by-nc-nd/4.0/). 2666-061X/19556

https://doi.org/10.1016/j.asmr.2020.04.011 
Arthroscopic partial meniscectomy may provide patients pain relief and return to a preinjury level of function. ${ }^{8}$

Despite focused indications for managing meniscal injuries as well as advancements of arthroscopic meniscectomy, the efficacy of the procedure in certain patient populations continues to remain debatable. ${ }^{9}$ Several randomized controlled trials have shown that meniscectomy provides functional outcomes equivalent to sham surgery or physical therapy. ${ }^{10-17}$ These investigations demonstrate that arthroscopic partial meniscectomy for degenerative tears with concomitant osteoarthritis provide no statistically significant improvement in patient-reported outcome measures in comparison to physical therapy or sham surgery. However, these randomized controlled trials possess several important limitations, such as the lack of patients with mechanical symptoms and high rates of crossover to the meniscectomy group. Furthermore, heterogeneous patient populations may have led to a nonsignificant difference in patient-reported outcome measures. Despite this, arthroscopic partial meniscectomy continues to remain a treatment option in meniscal pathology with adequate outcomes. ${ }^{5,7,18-22}$

The incidence of total knee arthroplasty (TKA) due to end-stage osteoarthritis is disproportionately increasing in younger patients. ${ }^{23,24}$ Furthermore, previous meniscectomy is an independent predictor of dissatisfaction following TKA. ${ }^{25}$ Because of the morbidity associated with multiple operations, it is imperative to identify the rate and risk factors associated with early reoperation or conversion to TKA.

The purpose of this investigation was to identify the rates and risk factors of revision arthroscopy and conversion to TKA within 1 year of isolated meniscectomy. We hypothesize that (1) there is a higher incidence of early conversion to TKA than to revision arthroscopy; (2) younger age and obesity are risk factors for revision arthroscopy; and (3) older age, obesity and concomitant osteoarthritis are risk factors for conversion to TKA.

\section{Methods}

\section{Database}

This was a retrospective review of the Humana and Medicare patient data within the PearlDiver Patient Records Database (PearlDiver, Fort Wayne, IN). The Humana data set contains approximately 20 million patients from 2007 through the second quarter of 2017, whereas the Medicare database includes 55 million insured patients from 2005 through 2014 and is from the $100 \%$ Standard Analytic Files. The accessed data represent procedures and diagnoses that were billed to the insurance company by the provider during that period. The PearlDiver database contains information regarding patient demographics, hospitalization details, diagnoses, procedures, and reimbursement. All data can be accessed with International Classification of Diseases, Ninth Revision (ICD-9) Procedural Codes, International Classification of Diseases, Tenth Revision (ICD-10) Procedural Codes, as well as Current Procedural Terminology (CPT) codes. All information in this database is deidentified and anonymous; therefore, this investigation was exempt from institutional review board approval.

\section{Study Groups}

The Humana and Medicare databases were queried for patients who underwent isolated meniscectomy (CPT-29880, CPT-29881) with an ICD-9 or ICD-10 diagnosis code of meniscal tear in their record on the same day of surgery (Table 1). Patients with bilateral procedures were counted as 2 separate procedures with laterality modifiers; however, patients with multiple meniscectomies were counted a single time to avoid overlapping data. Patients from all age groups were included. Patients with meniscal repair, concomitant ligamentous (anterior cruciate ligament, posterior cruciate ligament, medial collateral ligament, left collateral ligament, medial patellofemoral ligament) reconstruction or osteotomy (high tibial osteotomy, distal femoral osteotomy, triple tibial osteotomy) on the same day of meniscectomy and patients without a laterality modifier were excluded. Patients undergoing concomitant chondral procedures were included in this investigation.

\section{Study Outcomes}

Patients undergoing subsequent revision arthroscopy (29880, 29881, 29877, 29879), or ipsilateral TKA (CPT27447, ICD-9 Procedure Code 815.4) within 1 year after the date of isolated meniscectomy were identified via CPT or ICD-9 procedural codes (Table 1).

Table 1. CPT and ICD 9/10 Codes

\begin{tabular}{ll}
\hline \multicolumn{1}{c}{ Procedure } & \multicolumn{1}{c}{ CPT Codes } \\
\hline Meniscectomy & 29880,29881 \\
Chondroplasty & 29877,29879 \\
Total knee arthroplasty & 27447, 815.4 (ICD-9 Procedural \\
& Code) \\
Diagnosis & ICD-9/ICD-10 Codes \\
Meniscal tear & $836.1,836.0,717.5,717.49,717.43$, \\
& $717.42,717.41,717.40,717.14$, \\
& $717.3,717.2,717.1,717.0$, \\
& S83.289, S83.279, S83.269, \\
& S83.259, S83.249, S83.239, \\
& S83.229, S83.219, S83.209, \\
& S83.205, S83.202 \\
\hline
\end{tabular}

CPT, Current Procedural Terminology; ICD-9, International Classification of Disease, Ninth Revision; ICD-10, International Classification of Disease, Tenth Revision. 


\section{Statistical Analysis}

Patient demographics and comorbidities were queried by the database and included age, gender, obesity (30-39 $\left.\mathrm{kg} / \mathrm{m}^{2}\right)$, morbid obesity $\left(\geq 40 \mathrm{~kg} / \mathrm{m}^{2}\right)$, diabetes mellitus, tobacco use, alcohol use, hyperlipidemia, hypertension, coronary artery disease, chronic lung disease, chronic liver disease, chronic kidney disease, thyroid disease, depression, malnutrition, and knee osteoarthritis. Multivariate binomial logistical regression analysis was used to identify risk factors for subsequent ipsilateral reoperation or TKA within 1 year of the index isolated meniscectomy while controlling for knee osteoarthritis and all the aforementioned demographics and comorbidities. Odds ratios were calculated with $95 \%$ confidence intervals, and $P<0.05$ was considered statistically significant for all statistical comparisons.

\section{Results}

\section{Humana Database}

A total of 122,161 patients who underwent a meniscectomy were identified in the database, of whom $106,813(87.4 \%)$ patients contained laterality modifiers in their medical records. A total of 360 patients $(0.34 \%)$ underwent concomitant ligamentous reconstruction or osteotomy on the day of meniscectomy and were excluded from the investigation. Of the remaining group, 13,142 patients $(12.3 \%)$ who underwent isolated meniscectomy due to a meniscal tear were identified. Demographic data and medical comorbidities are provided in Table 2. Following isolated meniscectomy, 395 patients $(3.01 \%)$ underwent revision arthroscopy, and 629 patients $(4.79 \%)$ underwent TKA within 1 year of the index procedure.

Patients $<20$ years $(\mathrm{OR}=2.64, P=0.022), 20-29$ years $(\mathrm{OR}=3.30, P=0.002), 40-49$ years $(\mathrm{OR}=3.80$, $P<0.001)$, or $50-59$ years $(\mathrm{OR}=1.99, P=0.027)$ of age at the time of surgery, and obese patients $(\mathrm{OR}=1.33, P=0.003)$ had significantly higher rates of revision arthroscopy within 1 year of the index procedure, whereas concomitant osteoarthritis $(\mathrm{OR}=0.73$, $P=0.003)$ was associated with a lower risk of revision arthroscopy within 1 year of the index procedure (Table 3). Furthermore, tobacco use $(\mathrm{OR}=1.35$, $P=0.002)$, obesity $(\mathrm{OR}=1.64, P<0.001)$, hypertension $(\mathrm{OR}=1.30, P=0.009)$, and concomitant osteoarthritis $(\mathrm{OR}=1.42, P<0.001)$ were risk factors for TKA within 1 year of isolated meniscectomy.

\section{Medicare Database}

A total of 728,610 patients who underwent a meniscectomy were identified in the database, of whom $612,281(84.0 \%)$ patients contained a laterality modifier in their medical records. A total of 2,449 patients $(0.40 \%)$ underwent concomitant ligamentous repair or osteotomy on the day of meniscectomy and were excluded from the investigation. Of the remaining group, 407,888 patients $(66.9 \%)$ underwent isolated meniscectomy due to a meniscal tear. Demographic data and medical comorbidities are provided in Table 4. Following isolated meniscectomy, 3,770 patients $(0.92 \%)$ underwent revision arthroscopy, whereas 38,630 patients $(9.47 \%)$ underwent TKA within 1 year of the index procedure.

Patients $<64$ years of age at the time of surgery $(\mathrm{OR}=1.71 P<0.001)$ and with obesity $(\mathrm{OR}=1.10, P$ $<0.001)$, tobacco use (OR $=1.21, P<0.001)$ or depression $(\mathrm{OR}=1.39, P<0.001)$ were found to have risk factors for revision arthroscopy within 1 year of the index procedure. Concomitant osteoarthritis $(\mathrm{OR}=0.78, P<0.001)$, however, was associated with a lower risk of revision arthroscopy within 1 year of the index procedure (Table 5). Furthermore, patients 70-74 years $(\mathrm{OR}=1.12, P<0.001), 75-79$ years $(\mathrm{OR}=1.25$, $P<0.001$ ) or $80-85$ years (OR $=1.20, P<0.001$ ) of age at the time of surgery and obesity $(\mathrm{OR}=1.37$, $P<0.001)$, morbid obesity ( $\mathrm{OR}=1.20, P<0.001)$ or concomitant osteoarthritis $(\mathrm{OR}=1.46, P<0.001)$ were risk factors for TKA within 1 year of isolated meniscectomy. Age $<64$ years (OR $=0.69, P<0.001)$, age $>85$ years $(\mathrm{OR}=0.82, P<0.001)$ and diabetes mellitus $(\mathrm{OR}=0.89, P<0.001)$ were associated with lower risks of TKA within 1 year of isolated meniscectomy.

\section{Discussion}

The results of the present investigation demonstrate that $0.92 \%-3.01 \%$ of patients undergo revision arthroscopy, whereas $4.79 \%-9.47 \%$ of patients undergo TKA within 1 year following isolated meniscectomy. Obesity was an independent risk factor for earlyrevision arthroscopy and conversion to TKA. Patients younger than 60 years (except 30-39 years) in the Humana database and those younger than 64 in the Medicare database had higher risk of revision arthroscopy. Within the Medicare database, patients older than 70 years had an increased risk of conversion to TKA within 1 year of arthroscopic partial meniscectomy. Concomitant osteoarthritis was significantly associated with a lower risk of revision arthroscopy, but it was a risk factor for conversion to TKA in both databases. The findings of this investigation highlight variables that may increase the risk of failure of isolated meniscectomy.

Obesity $\left(30-39 \mathrm{~kg} / \mathrm{m}^{2}\right)$ was a risk factor for both early revision arthroscopy and conversion to TKA; however, morbid obesity $\left(\geq 40 \mathrm{~kg} / \mathrm{m}^{2}\right)$ was not a risk factor for early revision arthroscopy. Physicians may want to avoid further arthroscopic intervention in morbidly obese patients because they may experience a higher rate of complications following knee arthroscopy. ${ }^{26,27}$ Therefore, revision arthroscopy might not be offered 
Table 2. Meniscectomy Group Demographics, Humana Database

\begin{tabular}{lrr}
\hline \multicolumn{1}{c}{ Demographic } & $\mathrm{n}$ & \multicolumn{1}{c}{$\%$} \\
\hline Female & 7,148 & 54.4 \\
Male & 5,994 & 45.6 \\
Age, years & & \\
$\quad<20$ & 226 & 1.7 \\
$20-29$ & 297 & 2.3 \\
$30-39$ & 623 & 4.7 \\
$40-49$ & 1,681 & 12.8 \\
$50-59$ & 2,827 & 21.5 \\
$60-69$ & 4,135 & 31.5 \\
$70-79$ & 2,818 & 21.4 \\
$\quad>80$ & 535 & 4.1 \\
Obesity (30-39 kg/m $\left.{ }^{2}\right)$ & 4,012 & 30.5 \\
Morbid obesity $\left(\geq 40 \mathrm{~kg} / \mathrm{m}^{2}\right)$ & 1,749 & 13.3 \\
Tobacco use & 2,114 & 16.1 \\
Alcohol abuse & 294 & 2.2 \\
Diabetes mellitus & 4,118 & 31.3 \\
Hyperlipidemia & 9,477 & 72.1 \\
Hypertension & 9,204 & 70.0 \\
Coronary artery disease & 3,285 & 24.9 \\
Chronic kidney disease & 1,831 & 13.9 \\
Chronic liver disease & 1,362 & 10.4 \\
Chronic lung disease & 3,249 & 24.7 \\
Thyroid disease & 1,915 & 14.6 \\
Depression & 3,603 & 27.4 \\
Malnutrition & 274 & 2.1 \\
Osteoarthritis & 3,064 & \\
\hline & & \\
\hline & &
\end{tabular}

to patients with morbid obesity due to a theoretical increased risk of additional complications and failure. Morbid obesity was a risk factor for conversion to TKA in the Medicare database but not the Humana database. This may be due to varying patient demographics and patient selection in the Medicare and Humana databases. Despite the findings of this investigation, the effects of obesity on outcomes following meniscectomy are controversial because there is evidence to suggest that obesity portends worse outcomes, ${ }^{22,28,29}$ whereas some studies illustrate no relationship between obesity and outcomes. ${ }^{30}$ Based on the results of this investigation, obesity is a risk factor for additional surgery following meniscectomy; however, the decision to pursue revision arthroscopy or TKA is likely to be predicated on multiple factors. Nonetheless, physicians may counsel patients preoperatively that obesity may contribute to treatment failure, and surgeons may encourage patients to optimize their body mass indexes prior to the index procedure. Furthermore, diabetes and malnutrition were not risk factors for revision arthroscopy or conversion to TKA. Diabetes has been associated with being a risk factor for complications following meniscectomy. ${ }^{31-33}$ However, evidence that establishes malnutrition as a risk factor for complications following arthroscopic meniscectomy is limited. Patients' malnourished status may be reflected in their American Society of Anesthesiologists score, which has been shown to be a risk factor for complications following meniscectomy. ${ }^{31-33}$ The rationale for these variables' not being risk factors for additional surgery is not clearly evident; however, physicians may elect that patients to optimize their nutritional status and diabetes prior to further intervention so as to optimize outcomes.

In this investigation, depression was a risk factor for revision arthroscopy and conversion to TKA in the Medicare database, whereas it was a risk factor for conversion to TKA in the Humana database. The impact of psychological factors on outcomes following operative intervention in orthopaedics has been increasingly investigated. With respect to anterior cruciate ligament reconstruction, psychological factors lead to a decreased rate of return to sport and an increased length of time until return to sport. ${ }^{34}$ The most commonly cited

Table 3. Predictive Factors of Revision Arthroscopy or Total Knee Arthroplasty: Humana Database

\begin{tabular}{|c|c|c|c|c|}
\hline Variable & \multicolumn{2}{|c|}{ Revision Arthroscopy } & \multicolumn{2}{|c|}{ Total Knee Arthroplasty } \\
\hline Male sex & $0.99(0.84-1.17)$ & 0.91 & $0.90(0.79-1.02)$ & 0.92 \\
\hline$<20$ & $2.64(1.17-6.10)$ & 0.022 & $0.00(0.00-0.00)$ & 0.94 \\
\hline $20-29$ & $3.30(1.59-7.14)$ & 0.002 & $0.00(0.00-0.00)$ & 0.93 \\
\hline $30-39$ & $1.64(0.81-3.50)$ & 0.18 & $0.08(0.02-0.19)$ & $<0.001$ \\
\hline $40-49$ & $3.80(2.13-7.42)$ & $<0.001$ & $0.40(0.27-0.58)$ & $<0.001$ \\
\hline $60-69$ & $1.72(0.98-3.30)$ & 0.074 & $0.99(0.77-1.32)$ & 0.99 \\
\hline $70-79$ & $1.71-0.98-3.28)$ & 0.081 & $1.13(0.87-1.49)$ & 0.37 \\
\hline$>80$ & $\mathrm{~N} / \mathrm{A}$ & $\mathrm{N} / \mathrm{A}$ & $\mathrm{N} / \mathrm{A}$ & $\mathrm{N} / \mathrm{A}$ \\
\hline Obesity $\left(30-39 \mathrm{~kg} / \mathrm{m}^{2}\right)$ & $1.33(1.10-1.60)$ & 0.003 & $1.64(1.43-1.87)$ & $<0.001$ \\
\hline Morbid obesity $\left(\geq 40 \mathrm{~kg} \mathrm{~m}^{2}\right)$ & $0.81(0.62-1.04)$ & 0.11 & $1.17(0.99-1.37)$ & 0.058 \\
\hline Tobacco use & $1.15(0.93-1.42)$ & 0.19 & $1.35(1.15-1.57)$ & 0.002 \\
\hline Diabetes mellitus & $0.92(0.75-1.12)$ & 0.38 & $0.79(0.69-0.90)$ & 0.004 \\
\hline
\end{tabular}

NOTE. Boldface indicates statistical significance. 
Table 4. Meniscectomy Group Demographics: Medicare Database

\begin{tabular}{lcc}
\hline \multicolumn{1}{c}{ Demographic } & $\mathrm{n}$ & $\%$ \\
\hline Female & 245,147 & 60.1 \\
Male & 158,162 & 38.8 \\
Age, years & & \\
$\quad<64$ & 80,007 & 19.6 \\
$65-69$ & 141,659 & 34.7 \\
$70-74$ & 92,238 & 22.6 \\
$75-79$ & 55,634 & 13.6 \\
$80-84$ & 25,220 & 6.3 \\
$>85$ & 8,817 & 2.2 \\
Obesity (30-39 kg/m $\left.{ }^{2}\right)$ & 96,513 & 23.7 \\
Morbid obesity $\left(\geq 40 \mathrm{~kg} / \mathrm{m}^{2}\right)$ & 39,318 & 9.6 \\
Tobacco use & 70,330 & 17.2 \\
Alcohol abuse & 11,814 & 2.9 \\
Diabetes mellitus & 148,178 & 36.3 \\
Hyperlipidemia & 310,914 & 76.2 \\
Hypertension & 336,846 & 82.6 \\
Coronary artery disease & 146,224 & 35.8 \\
Chronic kidney disease & 61,160 & 15.0 \\
Chronic liver disease & 28,057 & 6.9 \\
Chronic lung disease & 92,913 & 22.8 \\
Thyroid disease & 338 & 0.08 \\
Depression & 113,343 & 27.8 \\
Malnutrition & 12,420 & 3.1 \\
Osteoarthritis & 85,478 & 21.0 \\
\hline
\end{tabular}

reason for the inability to return to sport is the fear of reinjury. ${ }^{34}$ Similarly, patients with depression and other psychological disorders may be fearful of returning to previous activities, and they experience lower functional improvement following arthroscopic meniscectomy. In turn, this may lead to a higher risk of revision arthroscopy or conversion to TKA due to continued symptomatology and pain.

Patients younger than 60 years typically were more likely to undergo early revision arthroscopy, and patients between the ages of 70 and 84 years in the Medicare database were more likely to undergo early conversion to TKA. It is important to note that age 3039 was not a risk factor for revision arthroscopy in the Humana database. The authors attribute this result to a statistical finding and not to a clinical reason. Furthermore, concomitant osteoarthritis was not a risk factor for early revision arthroscopy, whereas it was a risk factor for early conversion to TKA. The decision to pursue revision arthroscopy or TKA is likely to be a result of both age and osteoarthritis because older age is related to degree of osteoarthritis. ${ }^{35}$ Approximately $10 \%$ of patients undergoing meniscectomy require a TKA within 1 year of the initial meniscectomy. ${ }^{36,37}$ Barnds et al. demonstrated that arthroscopic partial meniscectomy survived 9 months before patients received TKA. ${ }^{36}$ In younger, more active patients with persistent symptoms following meniscectomy, surgeons may offer patients revision arthroscopy or other interventions, such as meniscal scaffolds, meniscal allograft transplantation or, in the case of advanced osteoarthritis, off-loading osteotomies or joint replacement. $^{38}$ Surgeons may be reluctant to offer TKA to younger patients because they face the risk of revision TKA at a younger age as well as the potential for multiple revision arthroplasties. Additionally, the proportion of meniscectomies performed in patients older than 50 years of age has decreased over time, ${ }^{39}$ which may be due to stringent insurance guidelines and reimbursement changes. ${ }^{39-41}$ Physicians and insurers may not pursue meniscectomy in a patient population that is likely to have advanced osteoarthritis and may soon undergo TKA. The presence of chondral injury predicts worse outcomes following isolated meniscectomy. ${ }^{22}$ If osteoarthritis was noted at the index procedure, physicians may be more likely to attribute persistent pain to the remaining arthritis and are unlikely to offer patients revision arthroscopy. Instead, more definitive treatment options such as TKA may be offered to patients with persistent symptoms.

Table 5. Predictive Factors of Revision Arthroscopy or Total Knee Arthroplasty: Medicare Database

\begin{tabular}{|c|c|c|c|c|}
\hline Variable & \multicolumn{2}{|c|}{ Revision Arthroscopy } & \multicolumn{2}{|c|}{ Total Knee Arthroplasty } \\
\hline Male sex & $1.02(0.97-1.07)$ & 0.46 & $0.85(0.84-0.86)$ & $<0.001$ \\
\hline$<64$ & $1.74(1.42-2.17)$ & $<0.001$ & $0.69(0.64-0.74)$ & $<0.001$ \\
\hline $65-69$ & $0.90(0.73-1.12)$ & 0.33 & $1.01(0.95-1.09)$ & 0.69 \\
\hline $70-74$ & $0.72(0.58-0.90)$ & 0.003 & $1.12(1.05-1.20)$ & $<0.001$ \\
\hline $75-79$ & $0.64(0.52-0.80)$ & $<0.001$ & $1.25(1.17-1.34)$ & $<0.001$ \\
\hline Obesity $\left(30-39 \mathrm{~kg} / \mathrm{m}^{2}\right)$ & $1.10(1.04-1.16)$ & $<0.001$ & $1.37(1.35-1.40)$ & $<0.001$ \\
\hline Morbid obesity $\left(\geq 40 \mathrm{~kg} / \mathrm{m}^{2}\right)$ & $1.01(0.94-1.09)$ & 0.71 & $1.20(1.17-1.23)$ & $<0.001$ \\
\hline Tobacco use & $1.21(1.14-1.28)$ & $<0.001$ & $1.01(0.99-1.03)$ & 0.50 \\
\hline Depression & $1.39(1.32-1.47)$ & $<0.001$ & $1.38(1.36-1.41)$ & $<0.001$ \\
\hline Malntrition & $1.03(0.91-1.16)$ & 0.60 & $0.90(0.87-0.94)$ & $<0.001$ \\
\hline Diabetes mellitus & $0.92(0.88-0.97)$ & 0.002 & $0.89(0.88-0.91)$ & $<0.001$ \\
\hline
\end{tabular}

NOTE. Boldface indicates statistical significance. 


\section{Limitations}

The analysis of this investigation must be interpreted within the context of the study's limitations. The main limitation of this study was that the degree of osteoarthritis was unknown. Therefore, patients with a small degree of degenerative changes are included with those who had severe osteoarthritis. This is a significant confounding variable in the investigation. The study used a national database of a single insurer; however, the quality and accuracy of the data rely on the procedural coding by physicians. Any miscoding or noncoding by providers is a potential source of error in these data. Thus, our reported revision rates may be an underestimation of the actual rates. Although this investigation included data from a Medicare database and Humana database, they may not be representative of the population of patients undergoing isolated meniscectomy. Factors that may influence early revision arthroscopy or conversion to TKA, such as indication for the procedure, amount of resected meniscus, laterality (medial or lateral), morphology of tear pattern (traumatic or degenerative tear), or degree of concomitant osteoarthritis, were unable to be queried. Patient factors that may explain revision surgery, such as level of activity intensity, occupational intensity or workers' compensation status were unknown and may be confounding factors in this investigation. Last, additional interventions for failed meniscectomy, such as meniscal scaffolds, meniscal allograft transplantation, cartilage restoration procedures, or osteotomies, may have been performed and were not captured and analyzed. Thus, the rate of reoperation is likely to be higher than reported in this investigation.

\section{Conclusions}

A Medicare and Humana database showed that $0.92 \%-3.01 \%$ and $4.79 \%-9.47 \%$ of patients undergo revision arthroscopy or conversion to TKA within 1 year of isolated meniscectomy. Obesity was a risk factor for early revision arthroscopy and conversion to TKA, whereas concomitant osteoarthritis was a risk factor for conversion to TKA.

\section{References}

1. Hutchinson ID, Moran CJ, Potter HG, Warren RF, Rodeo SA. Restoration of the meniscus: form and function. Am J Sports Med 2014;42:987-998.

2. Salata MJ, Gibbs AE, Sekiya JK. A systematic review of clinical outcomes in patients undergoing meniscectomy. Am J Sports Med 2010;38:1907-1916.

3. Ridley TJ, McCarthy MA, Bollier MJ, Wolf BR, Amendola A. Age differences in the prevalence of isolated medial and lateral meniscal tears in surgically treated patients. Iowa Orthop J 2017;37:91-94.

4. Losina E, Dervan EE, Paltiel AD, et al. Defining the value of future research to identify the preferred treatment of meniscal tear in the presence of knee osteoarthritis. PLoS One 2015;10:e130256.

5. Thorlund JB, Hare KB, Lohmander LS. Large increase in arthroscopic meniscus surgery in the middle-aged and older population in Denmark from 2000 to 2011. Acta Orthop 2014;85:287-292.

6. Hawker G, Guan J, Judge A, Dieppe P. Knee arthroscopy in England and Ontario: Patterns of use, changes over time, and relationship to total knee replacement. J Bone Joint Surg Am 2008;90:2337-2345.

7. Abrams GD, Frank RM, Gupta AK, Harris JD, McCormick FM, Cole BJ. Trends in meniscus repair and meniscectomy in the United States, 2005-2011. Am J Sports Med 2013;41:2333-2339.

8. Rao AJ, Erickson BJ, Cvetanovich GL, Yanke AB, Bach BR Jr, Cole BJ. The meniscus-deficient knee: Biomechanics, evaluation, and treatment options. Orthop J Sports Med 2015;3:2325967115611386.

9. Amin NH, Hussain W, Ryan J, Morrison S, Miniaci A, Jones MH. Changes within clinical practice after a randomized controlled trial of knee arthroscopy for osteoarthritis. Orthop J Sports Med 2017;5:2325967117698439.

10. Hare KB, Stefan Lohmander L, Kise NJ, Risberg MA, Roos EM. Middle-aged patients with an MRI-verified medial meniscal tear report symptoms commonly associated with knee osteoarthritis. Acta Orthop 2017;88: 664-669.

11. Moseley JB, O'Malley K, Petersen NJ, et al. A controlled trial of arthroscopic surgery for osteoarthritis of the knee. N Engl J Med 2002;347:81-88.

12. Lee DJ, Elfar JC. Utility of arthroscopic surgery for osteoarthritis of the knee. Geriatr Orthop Surg Rehabil 2015;6:47-49.

13. Kirkley A, Birmingham TB, Litchfield RB, et al. A randomized trial of arthroscopic surgery for osteoarthritis of the knee. N Engl J Med 2008;359:1097-1107.

14. Katz JN, Losina E. Surgery versus physical therapy for meniscal tear and osteoarthritis. N Engl J Med 2013;369: 677-678.

15. Katz JN, Wright J, Spindler KP, et al. Predictors and outcomes of crossover to surgery from physical therapy for meniscal tear and osteoarthritis: A randomized trial comparing physical therapy and surgery. J Bone Joint Surg Am 2016;98:1890-1896.

16. Sihvonen R, Paavola M, Malmivaara A, et al. Arthroscopic partial meniscectomy versus sham surgery for a degenerative meniscal tear. N Engl J Med 2013;369: 2515-2524.

17. Yim JH, Seon JK, Song EK, et al. A comparative study of meniscectomy and nonoperative treatment for degenerative horizontal tears of the medial meniscus. Am J Sports Med 2013:41:1565-1570.

18. Jarvinen TL, Sihvonen R, Englund M. Arthroscopy for degenerative knee-a difficult habit to break? Acta Orthop 2014;85:215-217.

19. Lubowitz JH, Provencher MT, Rossi MJ. Could the New England Journal of Medicine be biased against arthroscopic knee surgery? Part 2. Arthroscopy 2014;30:654-655.

20. Rossi MJ, D’Agostino RB Jr, Provencher MT, Lubowitz JH. Could the New England Journal of Medicine be biased against arthroscopic knee surgery? Arthroscopy 2014;30: 536-537. 
21. Price A, Beard D. Arthroscopy for degenerate meniscal tears of the knee. BMJ 2014;348:g2382.

22. Sofu H, Oner A, Camurcu Y, Gursu S, Ucpunar H, Sahin V. Predictors of the clinical outcome after arthroscopic partial meniscectomy for acute trauma-related symptomatic medial meniscal tear in patients more than 60 years of age. Arthroscopy 2016;32:1125-1132.

23. Kurtz SM, Lau E, Ong K, Zhao K, Kelly M, Bozic KJ. Future young patient demand for primary and revision joint replacement: National projections from 2010 to 2030. Clin Orthop Relat Res 2009;467:2606-2612.

24. Stambough JB, Clohisy JC, Barrack RL, Nunley RM, Keeney JA. Increased risk of failure following revision total knee replacement in patients aged 55 years and younger. Bone Joint J 2014;96-B:1657-1662.

25. Scott CE, Oliver WM, MacDonald D, Wade FA, Moran M, Breusch SJ. Predicting dissatisfaction following total knee arthroplasty in patients under 55 years of age. Bone Joint J 2016;98-B:1625-1634.

26. Sing DC, Luan TF, Feeley BT, Zhang AL. Is obesity a risk factor for adverse events after knee arthroscopy? Arthroscopy 2016;32:1346-1353, el341.

27. Nicolay RW, Selley RS, Terry MA, Tjong VK. Body mass index as a risk factor for 30-day postoperative complications in knee, hip, and shoulder arthroscopy. Arthroscopy 2019;35:874-882, e873.

28. Harrison MM, Morrell J, Hopman WM. Influence of obesity on outcome after knee arthroscopy. Arthroscopy 2004;20:691-695.

29. Spahn G, Muckley T, Kahl E, Hofmann GO. Factors affecting the outcome of arthroscopy in medialcompartment osteoarthritis of the knee. Arthroscopy 2006;22:1233-1240.

30. Kluczynski MA, Marzo JM, Wind WM, et al. The effect of body mass index on clinical outcomes in patients without radiographic evidence of degenerative joint disease after arthroscopic partial meniscectomy. Arthroscopy 2017;33: 2054-2063. e2010.

31. Gowd AK, Liu JN, Bohl DD, et al. Operative Time as an independent and modifiable risk factor for short-term complications after knee arthroscopy. Arthroscopy 2019;35:2089-2098.
32. Degen RM, Lebedeva Y, Birmingham TB, et al. Trends in knee arthroscopy utilization: A gap in knowledge translation. Knee Surg Sports Traumatol Arthrosc 2020;28: 439-447.

33. Basques BA, Gardner EC, Varthi AG, et al. Risk factors for short-term adverse events and readmission after arthroscopic meniscectomy: Does age matter? Am J Sports Med 2015:43:169-175.

34. Nwachukwu BU, Adjei J, Rauck RC, et al. How much do psychological factors affect lack of return to play after anterior cruciate ligament reconstruction? A systematic review. Orthop J Sports Med 2019;7:2325967119845313.

35. Hunter DJ. Risk stratification for knee osteoarthritis progression: A narrative review. Osteoarthrit Cartil 2009;17: 1402-1407.

36. Barnds B, Morris B, Mullen S, Schroeppel JP, Tarakemeh A, Vopat BG. Increased rates of knee arthroplasty and cost of patients with meniscal tears treated with arthroscopic partial meniscectomy versus non-operative management. Knee Surg Sports Traumatol Arthrosc 2019;27:2316-2321.

37. Abram SGF, Judge A, Beard DJ, Price AJ. Rates of knee arthroplasty within one year of undergoing arthroscopic partial meniscectomy in England: Temporal trends, regional and age-group variation in conversion rates. Osteoarthrit Cartil 2019;27:1420-1429.

38. Drobnic M, Ercin E, Gamelas J, et al. Treatment options for the symptomatic post-meniscectomy knee. Knee Surg Sports Traumatol Arthrosc 2019;27:1817-1824.

39. Essilfie A, Kang HP, Mayer EN, Trasolini NA, Alluri RK, Weber AE. Are orthopaedic surgeons performing fewer arthroscopic partial meniscectomies in patients greater than 50 years old? A national database study. Arthroscopy 2019;35:1152-1159, el151.

40. Potts A, Harrast JJ, Harner CD, Miniaci A, Jones MH. Practice patterns for arthroscopy of osteoarthritis of the knee in the United States. Am J Sports Med 2012;40: 1247-1251.

41. Holmes R, Moschetti W, Martin B, Tomek I, Finlayson S. Effect of evidence and changes in reimbursement on the rate of arthroscopy for osteoarthritis. Am J Sports Med 2013;41:1039-1043. 